\title{
Total distance, Wiener index and opportunity index in wreath products of star graphs
}

\author{
Matteo Cavaleri Alfredo Donno Andrea Scozzari \\ Università Niccolò Cusano \\ Rome, Italy \\ \{matteo.cavaleri, alfredo.donno, andrea.scozzari\}@unicusano.it
}

Submitted: Aug 7, 2018; Accepted: Jan 18, 2019; Published: Feb 8, 2019

(C) The authors. Released under the CC BY-ND license (International 4.0).

\begin{abstract}
In the last decades much attention has turned towards centrality measures on graphs. The Wiener index and the total distance are key tools to investigate the median vertices, the distance-balanced property and the opportunity index of a graph. This interest has recently been addressed to graphs obtained via classical graph products like the Cartesian, the direct, the strong and the lexicographic product. We extend this study to a relatively new graph product, that is, the wreath product. In this paper, we compute the total distance for the vertices of an arbitrary wreath product graph $G \geq H$ in terms of the total distances in $H$ and of some distance-based indices of $G$. We explicitly compute these indices for the star graph $S_{n}$, providing a closed formula for the total distances in $S_{n}$ 乙 $H$ when $H$ is complete or a star. As a consequence, we obtain the Wiener index of these graphs, we characterize the median and the central vertices, and finally we give an upper and a lower bound for the opportunity index of $S_{n} \prec S_{m}$ in terms of tail conditional expectations of an associated binomial distribution.
\end{abstract}

Mathematics Subject Classifications: 05C12, 05C57, 05C76

\section{Introduction}

Centrality measures on graphs have represented a long standing interest for researchers both from a theoretical and an application viewpoint. An interesting example is the placing of one or more facilities on a network, that is, given a set of clients that has to be served by the facilities, the aim is to find a location for the facilities that optimizes certain centrality criteria. The classical measures of the centrality of a graph include the center and the median. These criteria allow to find subsets of vertices with specific characteristics and properties $[17,18]$. These concepts were naturally generalized to some other measures such as the centdian, the branch weight centroid of a graph, the leaf weight 
median, the leaf branch weight centroid, the distance balance center, the $k$-centrum and so forth. Reid [25] gives a thorough survey of various concepts that have been defined and studied as a measure of central substructure in a tree. Anyhow, all these measures are based on the concept of distances or of average distances between vertices in a given graph, which is strictly related to the classical Wiener index of a connected graph, defined as the sum of the distances between all pairs of vertices [28]. This index was extensively studied in the last decades on several classes of graphs. Bounds on the average distance for trees, cycles, and graphs with minimum degree at least 2 were also provided in [10]. In [9] it is shown that every connected graph of order $n$ and minimum degree $\delta$ has a spanning tree with given bounded average distance.

The study of average distances is also strictly related to the problem of partitioning the vertex set of a graph. In particular, the notion of balanced bi-partition of the vertices of a tree has been introduced and studied by many authors (see, e.g., [24] and the references therein). The problem is to find a bi-partition of the vertex set of the tree such that the two sums of the distances from a given vertex $v$ to all the vertices in each of the two subtrees are as close as possible, where the two subtrees have only $v$ in common. This concept can be further extended to more general classes of graphs and leads to the definition of distance-balanced graph (see Section 2 for a formal definition). Distancebalanced graphs were introduced in [21], where the authors studied some basic properties and local operations. Cabello and Lukšič considered the problem of finding the minimum number of edges that can be added to a given graph to obtain a distance-balanced graph [6]. They proved that the problem is NP-hard for graphs of diameter 3, but can be solved in polynomial time for graphs of diameter 2. Distance-balanced graphs find a natural application in Game Theory and Strategic Interaction models. Actually, in [10] the author considers a situation when there are two firms competing in a common market with an objective to minimize the cost of transport between all the vertices (clients) of the network. It is desirable to design a network in such a way that its vertex set can be divided into two subsets so that neither of the two involved firms has an advantage to the other likewise reducing the cost of distribution and transportation of goods [3]. Another interesting application is in Social Network Analysis, where one wants to identify (connected) clusters of members with homogenous features. In particular, in the case of a bi-partition of the vertex set of a network, the minimum of the largest difference between the relative Wiener indices of the two subsets is an equity measure of the partition of the vertex set into two equal parts. The largest difference between the Wiener indices of two subsets is defined as the opportunity index of the graph. Equal opportunity graphs are precisely those graphs having the opportunity index equal to zero. Furthermore, in [3] the authors show that equal opportunity graphs are precisely distance-balanced graphs (of even order) that correspond to a class of graphs first studied in [16] in the case of partial cubes. Other interesting applications of distance-balanced graphs can be found in [19].

In this paper we study some centrality measures in the wreath product of graphs, focusing our attention to some special classes. The wreath product of graphs represents a graph analogue of the classical wreath product of groups [23]. In the literature (see, e.g., 
[21]), the relationship between total distance, opportunity index, and distance-balanced property have been considered for the classical compositions of graphs: the Cartesian, the direct, the strong, the lexicographic product. Moreover, we mention that in [22] the notion of strongly distance-balanced graph has been introduced, and it has been investigated in $[2,22]$ for graph compositions. The Wiener index of a wreath product has been studied in [7], and in [4, 13] for some special families of graphs. Spectral computations have been performed in $[5,13]$. However, to the best of our knowledge, the total distance, the opportunity index and distance-balanced property have never been investigated for wreath products. Hence, our paper starts this kind of investigation.

The total distance of a vertex in a graph is the sum of the distances between this vertex and all other vertices. This quantity characterizes the median vertices (vertices with minimal total distance), the distance-balanced property and the opportunity index of a graph (see [3]). In this paper, we compute the total distance of the vertices of a wreath product $G$ < $H$ in terms of the total distance of the vertices of $H$, and of some distancebased indices of $G$, specially defined for the wreath product but independent of the graph $H$. The metric structure of a wreath product is interesting because the computation of the distance between two vertices of $G$ ? $H$ involves the minimal length of a walk, in the graph $G$, with prescribed start and end vertices, and a subset of the vertices that the walk has to visit. This is strictly connected to the Travelling Salesman Problem, which is a well-known NP-hard optimization problem. Our study would open the possibility to investigate, with further intriguing developments, the distance-balanced property for the wreath product of graphs. Here, we focus our attention on a non-distance-balanced case, the star graph $S_{n}$, in order to obtain a non-trivial variability of the total distance. Our explicit computation of the above mentioned indices for the graph $S_{n}$ allows us to obtain a formula for the total distance of the vertices in the wreath product $\left.S_{n}\right\} H$, whenever the total distances in $H$ are known. We apply this formula to the case $S_{n} \prec K_{m}$, where $K_{m}$ is the complete graph on $m$ vertices, and to the case $S_{n} \imath S_{m}$. This analysis makes us able to detect the central and the median vertices, to compute the Wiener index and to obtain an upper and a lower bound for the opportunity index of $S_{n} 2 S_{m}$.

The paper is organized as follows. In Section 2, we provide some notation and recall the basic definition of the wreath product of two graphs (Definition 1). In particular, we present the known relations between total distance, distance-balanced property and opportunity index. In Section 3, we present some results and notation from [7] in order to prove the main results of the section, which are Theorem 17 and Corollary 19: they provide a formula for the total distance in $G ? H$ in terms of total distances in $H$ and of the indices $W_{\rho_{k}}(u, G)$ (see Definition 18). In Section 4, we give an explicit computation of the indices $W_{\rho_{k}}\left(u, S_{n}\right)$ (see Theorem 22). This makes us able to analyze the cases $S_{n}$ 乙 $K_{m}$ and $S_{n} 2 S_{m}$, presenting the total distances (Theorem 23 and Theorem 28), detecting the median vertices (Corollary 24 and Corollary 29) and computing the Wiener index (Corollary 25 and Corollary 32). Finally, we give in Theorem 38 some lower and upper bounds for the opportunity index of $S_{n} \prec S_{m}$, in terms of tail conditional expectations of the binomial distribution with probability $p=\frac{m-1}{m}$. 


\section{Preliminaries and motivations}

Every graph considered in this paper will be finite, connected, and simple, that is, loops and multiple edges are not admitted. Such a graph will be denoted by $G=\left(V_{G}, E_{G}\right)$, where $V_{G}$ denotes the vertex set, and $E_{G}$ is the edge set consisting of unordered pairs of type $\{u, v\}$, with $u, v \in V_{G}$. When $\{u, v\} \in E_{G}$, we say that the vertices $u$ and $v$ are adjacent in $G$, and we write $u \sim v$. A walk of length $\ell$ in $G$ is a sequence $u_{0}, u_{1}, \ldots, u_{\ell}$ of vertices such that $u_{i} \sim u_{i+1}$, for each $i=0, \ldots, \ell-1$. The walk is said to be closed if $u_{0}=u_{\ell}$. Since the graph $G$ is connected, for every $u, v \in V_{G}$, there exists a walk $u_{0}, u_{1}, \ldots, u_{\ell}$ in $G$ such that $u_{0}=u$ and $u_{\ell}=v$. For a graph $G$, we will denote by $d_{G}(u, v)$ the geodesic distance (or distance for short) between the vertices $u$ and $v$, that is, the length of a shortest walk in $G$ joining $u$ and $v$. The eccentricity of a vertex $u \in V_{G}$ is defined as $e_{G}(u)=\max _{v \in V_{G}}\left\{d_{G}(u, v)\right\}$ and $u$ is a center for $G$ if $e_{G}(u)=\min _{v \in V_{G}}\left\{e_{G}(v)\right\}$. The diameter of $G$ is then defined as $\operatorname{diam}(G)=\max _{u \in V_{G}}\left\{e_{G}(u)\right\}$. In particular, $\operatorname{diam}(G)<\infty$ as $G$ is connected. A vertex $u \in V_{G}$ has degree $k$ if there exist exactly $k$ vertices in $G$ adjacent to $u$. We will write $\operatorname{deg} u=k$. We say that $G$ is regular of degree $k$, or $k$-regular, if $\operatorname{deg} u=k$, for every $u \in V_{G}$.

We recall now the fundamental definition of the wreath product of graphs.

Definition 1. Let $G=\left(V_{G}, E_{G}\right)$ and $H=\left(V_{H}, E_{H}\right)$ be two graphs, with $\left|V_{G}\right|=n$. Let us fix an enumeration of the vertices of $G$ so that $V_{G}=\left\{x_{1}, x_{2}, \ldots, x_{n}\right\}$. The wreath product $G$ ? $H$ is the graph with vertex set $V_{H}^{V_{G}} \times V_{G}=\left\{\left(y_{1}, \ldots, y_{n}\right) x_{i} \mid y_{j} \in V_{H}, x_{i} \in V_{G}\right\}$, where two vertices $u=\left(y_{1}, \ldots, y_{n}\right) x_{i}$ and $v=\left(y_{1}^{\prime}, \ldots, y_{n}^{\prime}\right) x_{k}$ are connected by an edge if:

1. (edges of type $I$ ) either $i=k=: i_{*}$ and $y_{j}=y_{j}^{\prime}$ for every $j \neq i_{*}$, and $y_{i_{*}} \sim y_{i_{*}}^{\prime}$ in $H$;

2. (edges of type $I I)$ or $y_{j}=y_{j}^{\prime}$, for every $j=1, \ldots, n$, and $x_{i} \sim x_{k}$ in $G$.

It follows from Definition 1 that if $\left|V_{G}\right|=n$ and $\left|V_{H}\right|=m$, the graph $G$ ? $H$ has $n m^{n}$ vertices. It is easy to see that $G \prec H$ is connected if and only if $G$ and $H$ are connected. Moreover, if $G$ is a regular graph of degree $r_{G}$ and $H$ is a regular graph of degree $r_{H}$, then the graph $G$ ? $H$ is an $\left(r_{G}+r_{H}\right)$-regular graph.

Notice that, in the case $\left|V_{G}\right|=1$, the graph $G$ < $H$ is isomorphic to $H$; on the other hand, if $\left|V_{H}\right|=1$, then the graph $G$ 々 $H$ is isomorphic to $G$. In the rest of the paper, we assume $\left|V_{G}\right|>1$ and $\left|V_{H}\right|>1$.

It is a classical fact (see, for instance, [29]) that the simple random walk on the graph $G$ ? $H$ is the so called Lamplighter random walk, according to the following interpretation: suppose that at each vertex of $G$ (the base graph) there is a lamp, whose possible states (or colors) are represented by the vertices of $H$ (the color graph), so that the vertex $\left(y_{1}, \ldots, y_{n}\right) x_{i}$ of $G$ ? $H$ represents the configuration of the $n$ lamps at each vertex of $G$ (for each vertex $x_{j} \in V_{G}$, the lamp at $x_{j}$ is in the state $y_{j} \in V_{H}$ ), together with the position $x_{i}$ of a lamplighter walking on the graph $G$. At each step, the lamplighter may either go to a neighbor of the current vertex $x_{i}$ and leave all lamps unchanged (this situation corresponds to edges of type II in $G$ < $H$ ), or he may stay at the vertex $x_{i} \in G$, but he changes the state of the lamp which is in $x_{i}$ to a neighbor state in $H$ (this situation 
corresponds to edges of type I in $G$ 々 $H$ ).

It is worth mentioning that the wreath product of graphs represents a graph analogue of the classical wreath product of groups, as it turns out that the wreath product of the Cayley graphs of two finite groups is the Cayley graph of the wreath product of the groups, with a suitable choice of the generating sets. In the paper [12], this correspondence is proven in the more general context of generalized wreath products of graphs, inspired by the construction introduced in [1] for permutation groups. Also, observe that in [8] the wreath product of matrices has been defined, in order to describe the adjacency matrix of the wreath product of two graphs and to perform spectral computations.

We recall here the definitions of the four classical products, namely the Cartesian, the direct, the strong and the lexicographic product, of two graphs $G=\left(V_{G}, E_{G}\right)$ and $H=$ $\left(V_{H}, E_{H}\right)$. Observe that all the products have $V_{G} \times V_{H}$ as vertex set and are associative [15].

Definition 2. Let $G=\left(V_{G}, E_{G}\right)$ and $H=\left(V_{H}, E_{H}\right)$ be two finite graphs.

- The Cartesian product $G \square H$ is the graph where two vertices $(u, v)$ and $\left(u^{\prime}, v^{\prime}\right)$ are adjacent if $u \sim u^{\prime}$ in $G$ and $v=v^{\prime}$ in $H$, or if $v \sim v^{\prime}$ in $H$ and $u=u^{\prime}$ in $G$.

- The direct product $G \times H$ is the graph where two vertices $(u, v)$ and $\left(u^{\prime}, v^{\prime}\right)$ are adjacent if $u \sim u^{\prime}$ in $G$ and $v \sim v^{\prime}$ in $H$.

- The strong product $G \otimes H$ is the graph with edge set $E_{G \square H} \cup E_{G \times H}$.

- The lexicographic product $G \circ H$ is the graph where two vertices $(u, v)$ and $\left(u^{\prime}, v^{\prime}\right)$ are adjacent if $u \sim u^{\prime}$ in $G$, or $u=u^{\prime}$ in $G$ and $v \sim v^{\prime}$ in $H$.

Remark 3. Notice that the graph $G<H$ can be regarded as a spanning subgraph of $H^{\square n} \square G$, where $H^{\square n}$ denotes the iterated Cartesian product of $H$ with itself $n$ times. This embedding relates the geodesic distance in $H^{\square n}$ with the geodesic distance in $G$ 々 $H$, as we will point out in Theorem 13.

The study of the opportunity index, that we will develop in Section 4.2 for the wreath product of two star graphs, is strictly related to the property of a graph of being distancebalanced. For this reason, we recall here some basic definitions. Distance-balanced graphs were introduced in [21], where the authors studied some basic properties, local operations, and the behavior of graph products with respect to this property.

Let $G=\left(V_{G}, E_{G}\right)$ be a graph, and let $u, v \in V_{G}$ such that $u \sim v$. Then one defines:

$$
W_{u v}^{G}=\left\{z \in V_{G} \mid d_{G}(z, u)<d_{G}(z, v)\right\},
$$

that is, $W_{u v}^{G}$ is the set of vertices closer to $u$ than to $v$. Notice that the sets $W_{u v}^{G}$, with $u, v$ adjacent vertices in $G$, play also an important role in Chemical Graph Theory where, for instance, the Szeged index of the graph $G$ is defined as $S z(G)=\sum_{u \sim v} W_{u v}^{G} W_{v u}^{G}$ (see [27] for more details).

Definition 4. A graph $G=\left(V_{G}, E_{G}\right)$ is distance-balanced if $\left|W_{u v}^{G}\right|=\left|W_{v u}^{G}\right|$, for every pair of adjacent vertices $u, v \in V_{G}$. 
Cyclic graphs and complete graphs are simple examples of distance-balanced graphs. It is shown in [21] that a graph $G$ of diameter 2 is distance-balanced if and only if it is regular. Moreover, a vertex-transitive graph, that is a graph whose automorphism group acts transitively on the vertex set, is distance-balanced. On the other hand, it is known that there exist distance-balanced graphs which are not vertex-transitive (see, e.g., [20]).

It is proven in [21] that the Cartesian product $G \square H$ is distance-balanced if and only if both $G$ and $H$ are distance-balanced; moreover, the lexicographic product $G \circ H$ is distance-balanced if and only if $G$ is distance-balanced and $H$ is regular. On the other hand, it is shown there, by explicit counterexamples, that the direct product and the strong product do not preserve the property of being distance-balanced.

We also want to mention that in [22] the notion of strongly distance-balanced graph has been introduced, and it has been investigated in $[2,22]$ for graph compositions. However, the property of being distance-balanced has never been investigated for wreath products: our paper starts this kind of investigation.

Definition 5. Let $G=\left(V_{G}, E_{G}\right)$ be a connected graph and let $v \in V_{G}$. The total distance of $v$ is

$$
W(v, G)=\sum_{u \in V_{G}} d_{G}(v, u),
$$

that is, the sum of the distances of $v$ from each vertex of $G$. The median $M(G)$ of $G$ is the set of vertices of $G$ for which the value $W(v, G)$ is minimal among all vertices of $G$.

We will use in Section 3 the following characterization of distance-balanced graphs given in [2].

Proposition 6. A connected graph $G=\left(V_{G}, E_{G}\right)$ is distance-balanced if and only if $M(G)=V_{G}$.

It follows that for distance-balanced graphs the condition $W(u, G)=W(v, G)$ is satisfied for all vertices $u, v \in V_{G}$. In these graphs, vertices have the same relevance, but they are not necessarily indistinguishable, so they are of special interest in the study of social networks, as all people in such graphs are equal in some sense.

For any $U \subseteq V_{G}$, the relative Wiener index of $U$ is defined as

$$
W_{U}(G)=\sum_{\{u, v\} \in\left(\begin{array}{c}
U \\
2
\end{array}\right)} d_{G}(u, v),
$$

that is, $W_{U}(G)$ is the sum of the distances between all the (unordered) pairs of vertices in $U$. For $U=V_{G}$, one gets the classical Wiener index $W(G)=\frac{1}{2} \sum_{u, v \in V_{G}} d_{G}(u, v)$ of the graph $G$, introduced in [28]. Moreover, the following identity holds:

$$
W(G)=\frac{1}{2} \sum_{v \in V_{G}} W(v, G) .
$$

Following [3], we recall now the definition of equal opportunity graph. 
Let $G=\left(V_{G}, E_{G}\right)$ be a graph with an even number of vertices $2 n$, and let $V_{1}, V_{2}$ be two subsets of $V_{G}$ such that $V_{G}=V_{1} \sqcup V_{2}$ and $\left|V_{1}\right|=\left|V_{2}\right|=n$ (we denote by $\sqcup$ the disjoint union). If this is the case, we say that $\left\{V_{1}, V_{2}\right\}$ is a half-partition of $G$.

The opportunity index opp $(G)$ of a graph $G$ is then defined as

$$
\operatorname{opp}(G)=\max \left\{\left|W_{V_{1}}(G)-W_{V_{2}}(G)\right|:\left\{V_{1}, V_{2}\right\} \text { is a half-partition of } G\right\} .
$$

In other words, the opportunity index of $G$ is the largest possible difference between the relative Wiener indices of the two halves of $G$, when considering all partitions of its vertex set into two equal parts.

Definition 7. A graph $G=\left(V_{G}, E_{G}\right)$ is said to be an equal opportunity graph if $\operatorname{opp}(G)=$ 0 .

The following result is proven in [3].

Proposition 8. A graph $G=\left(V_{G}, E_{G}\right)$ is an equal opportunity graph if and only if it is a distance-balanced graph with an even number of vertices.

In particular, it follows that a vertex-transitive graph with an even number of vertices is an equal opportunity graph.

Example 9. Let us denote by $K_{n}=\left(V_{K_{n}}, E_{K_{n}}\right)$ the complete graph on $n$ vertices (in Fig. 1, the graph $K_{5}$ is represented). The complete graph is vertex-transitive and therefore distance-balanced. Hence, it is an equal opportunity graph when $n$ is even. The vertices are indistinguishable and then, for every $v \in V_{K_{n}}$ :

$$
W\left(v, K_{n}\right)=\sum_{u \in V_{K_{n}}} d_{G}(u, v)=n-1 .
$$

In particular, from Equation (2) we get

$$
W\left(K_{n}\right)=\frac{n(n-1)}{2} .
$$

Example 10. Let us denote by $S_{n}=\left(V_{S_{n}}, E_{S_{n}}\right)$ the star graph on $n \geqslant 3$ vertices (in Fig. 1, the graph $S_{5}$ is represented). The vertex set of the graph $S_{n}$ is $V_{S_{n}}=\{c\} \sqcup L$, with $|L|=n-1$, and the set of edges is $E_{S_{n}}=\{\{c, f\}: f \in L\}$. The element $c$ is called center and the elements in $L$ are called leaves. Then:

$$
W\left(v, S_{n}\right)= \begin{cases}n-1 & \text { if } v=c \\ 2 n-3 & \text { if } v \neq c .\end{cases}
$$

In particular, from Equation (2) we get

$$
W\left(S_{n}\right)=(n-1)^{2} .
$$

By virtue of Proposition 6, the graph $S_{n}$ is not distance-balanced. If $n=2 k$, with $k \in \mathbb{N}$, it is easy to see that $\operatorname{opp}\left(S_{n}\right)=k-1$. 

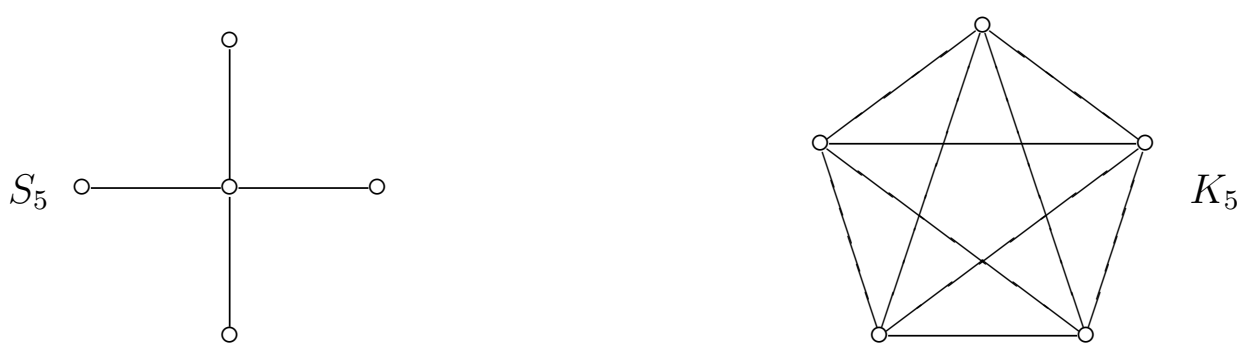

Figure 1: The graphs $S_{n}$ and $K_{n}$, for $n=5$.

\section{Distances in a wreath product}

In this section we are going to analyze the variability of the total distance in the vertices of the wreath product $G \imath H$. We start by recalling some definitions and results from [7], where a detailed study of the Wiener index of $G$ ? $H$ has been developed.

Definition 11. Let $G=\left(V_{G}, E_{G}\right)$ be a graph and let $A \subseteq V_{G}$. We define a map $\rho_{A}$ on $V_{G} \times V_{G}$ such that, for any pair of vertices $u$ and $v$ in $V_{G}$, the number $\rho_{A}(u, v)$ is the length of a shortest walk joining $u$ and $v$, visiting all vertices in $A$. When $A=V_{G}$, we write $d_{H a}:=\rho_{V_{G}}$, and $d_{H a}(u, v)$ is said the Hamiltonian distance of $u$ and $v$.

Observe that $\rho_{A}(u, v)=\rho_{A}(v, u)$, for each $A \subseteq V_{G}$ and $u, v \in V_{G}$.

Definition 12. Let $G=\left(V_{G}, E_{G}\right)$ be a graph. For any $u \in V_{G}$, the Hamiltonian eccentricity of the vertex $u$ is

$$
e_{H a}(u):=\max \left\{d_{H a}(u, v), v \in V_{G}\right\} .
$$

Similarly, the Hamiltonian diameter of $G$ is

$$
\operatorname{diam}_{H a}(G):=\max \left\{e_{H a}(u), u \in V_{G}\right\} .
$$

Recall that, fixed an ordering for the vertices of $G$, say $V_{G}=\left\{x_{1}, \ldots, x_{n}\right\}$, a vertex $u$ of $G$ 々 $H$ can be written as $u=\left(y, x_{i}\right)$, with $y \in\left(V_{H}\right)^{n}$ and $i \in\{1, \ldots, n\}$. The $n$-tuple $y$ in $\left(V_{H}\right)^{n}$ can be regarded as a vertex of the $n$-th Cartesian power of $H$ (see Definition 2). It is well known (see, e.g., [15]) that the distance in the Cartesian product is nothing but the sum of the distances, computed coordinatewise. In other words, given two vertices $y=\left(y_{1}, \ldots, y_{n}\right)$ and $y^{\prime}=\left(y_{1}^{\prime}, \ldots, y_{n}^{\prime}\right)$ in $V_{H^{\square n}}$, with $y_{i}, y_{i}^{\prime} \in V_{H}$ for each $i=1, \ldots, n$, one has:

$$
d_{H^{\square}}\left(y, y^{\prime}\right)=\sum_{i=1}^{n} d_{H}\left(y_{i}, y_{i}^{\prime}\right) .
$$

Consider now the graph $G$ 々 H. Then two vertices $y$ and $y^{\prime}$ of $V_{H^{\square n}}$ define the following subset of $V_{G}$ :

$$
\delta\left(y, y^{\prime}\right):=\left\{x_{i} \in V_{G}: y_{i} \neq y_{i}^{\prime}\right\}
$$


In the Lamplighter interpretation, $\delta\left(y, y^{\prime}\right)$ consists of the vertices of the base graph $G$ where the given lamp configurations $y$ and $y^{\prime}$ do not coincide.

Theorem 13. [7] Let $G=\left(V_{G}, E_{G}\right)$ and $H=\left(V_{H}, E_{H}\right)$ be two graphs. Suppose $\left|V_{G}\right|=n$. For any vertices $u=\left(y_{1}, \ldots, y_{n}\right) x, v=\left(y_{1}^{\prime}, \ldots, y_{n}^{\prime}\right) x^{\prime} \in G$ ? $H$, we have:

$$
d_{G \imath H}(u, v)=d_{H^{\square n}}\left(y, y^{\prime}\right)+\rho_{\delta\left(y, y^{\prime}\right)}\left(x, x^{\prime}\right)=\sum_{i=1}^{n} d_{H}\left(y_{i}, y_{i}^{\prime}\right)+\rho_{\delta\left(y, y^{\prime}\right)}\left(x, x^{\prime}\right),
$$

where $y=\left(y_{1}, \ldots, y_{n}\right)$ and $y^{\prime}=\left(y_{1}^{\prime}, \ldots, y_{n}^{\prime}\right)$.

Corollary 14. [7] For any $u=\left(y_{1}, \ldots, y_{n}\right) x \in V_{G l H}$ we have:

$$
e_{G \imath H}(u)=\sum_{i=1}^{n} e_{H}\left(y_{i}\right)+e_{H a}(x) .
$$

In particular:

$$
\operatorname{diam}(G \curlywedge H)=n \operatorname{diam}(H)+\operatorname{diam}_{H a}(G) .
$$

As for the investigation of the distances in [7], we start here our study of the total distance in $G$ ? $H$ by analyzing the Cartesian product $H^{\square n}$.

Lemma 15. Let $H=\left(V_{H}, E_{H}\right)$ be a graph, with $\left|V_{H}\right|=m$. Let $y=\left(y_{1}, \ldots, y_{n}\right) \in V_{H^{\square n}}$, with $n \geqslant 1$. Then we have

$$
W\left(y, H^{\square n}\right)=m^{n-1} \sum_{i=1}^{n} W\left(y_{i}, H\right) .
$$

Proof. Set $y^{\prime}=\left(y_{1}^{\prime}, \ldots, y_{n}^{\prime}\right)$, with $y_{i}^{\prime} \in V_{H}$ for each $i=1, \ldots, n$. Then

$$
W\left(y, H^{\square n}\right)=\sum_{y^{\prime} \in V_{H} \square n} d_{H^{\square n}}\left(y, y^{\prime}\right)=\sum_{y^{\prime} \in V_{H} \square n} \sum_{i=1}^{n} d_{H}\left(y_{i}, y_{i}^{\prime}\right)=\sum_{i=1}^{n} \sum_{y^{\prime} \in V_{H} \square n} d_{H}\left(y_{i}, y_{i}^{\prime}\right) .
$$

For a fixed $i$ and a fixed $u \in V_{H}$, the contribution $d_{H}\left(y_{i}, u\right)$ appears exactly $m^{n-1}$ times in the sum. Therefore we obtain:

$$
W\left(y, H^{\square n}\right)=\sum_{i=1}^{n} m^{n-1} \sum_{u \in V_{H}} d_{H}\left(y_{i}, u\right)=m^{n-1} \sum_{i=1}^{n} W\left(y_{i}, H\right) .
$$

Definition 16. Let $G=\left(V_{G}, E_{G}\right)$ be a graph, and let $u \in V_{G}$, and $A \subseteq V_{G}$. We put $W_{\rho_{A}}(u, G):=\sum_{v \in V_{G}} \rho_{A}(u, v)$.

Theorem 17. Let $G=\left(V_{G}, E_{G}\right)$ and $H=\left(V_{H}, E_{H}\right)$ be two graphs with $\left|V_{G}\right|=n$, $\left|V_{H}\right|=m$. Let $u=(y, x)=\left(y_{1}, \ldots, y_{n}\right) x \in V_{G i H}$. Then:

$$
W(u, G \curlywedge H)=n m^{n-1} \sum_{i=1}^{n} W\left(y_{i}, H\right)+\sum_{A \subseteq V_{G}}(m-1)^{|A|} W_{\rho_{A}}(x, G) .
$$


Proof. Let $u^{\prime}=\left(y^{\prime}, x^{\prime}\right)$ in $V_{G r H}$, with $y^{\prime} \in V_{H^{\square n}}$ and $x^{\prime} \in V_{G}$. Then, by Theorem 13, we have

$$
W(u, G \curlywedge H)=\sum_{u^{\prime} \in V_{G \backslash H}}\left(d_{H^{\square n}}\left(y, y^{\prime}\right)+\rho_{\delta\left(y, y^{\prime}\right)}\left(x, x^{\prime}\right)\right) .
$$

For a fixed $y^{\prime} \in H^{\square n}$, the contribution $d_{H^{\square n}}\left(y, y^{\prime}\right)$ appears $n$ times in the sum so that, using formula of Lemma 15, we get:

$$
\sum_{u^{\prime} \in V_{G \backslash H}} d_{H^{\square n}}\left(y, y^{\prime}\right)=n \sum_{y^{\prime} \in V_{H} \square n} d_{H^{\square n}}\left(y, y^{\prime}\right)=n W\left(y, H^{\square n}\right)=n m^{n-1} \sum_{i=1}^{n} W\left(y_{i}, H\right) .
$$

On the other hand, for any subset $A \subseteq V_{G}$, we have:

$$
\left|\left\{y^{\prime} \in V_{H^{\square n}}: \delta\left(y, y^{\prime}\right)=A\right\}\right|=(m-1)^{|A|},
$$

since the coordinates of $y^{\prime}$ outside $A$ must be equal to the corresponding coordinates of $y$, but we can choose $|A|$ coordinates of $y^{\prime}$ among the $m-1$ elements of $V_{H}$ (they have to be different from the ones of $y$ ). This implies that, for every $x^{\prime} \in V_{G}$ and any fixed $A \subseteq V_{G}$, the contribution given by $\rho_{A}\left(x, x^{\prime}\right)$ appears $(m-1)^{|A|}$ times in the sum, so that:

$$
\sum_{u^{\prime} \in V_{G \backslash H}} \rho_{\delta\left(y, y^{\prime}\right)}\left(x, x^{\prime}\right)=\sum_{A \subseteq V_{G}} \sum_{x^{\prime} \in V_{G}}(m-1)^{|A|} \rho_{A}\left(x, x^{\prime}\right) .
$$

Then the claim is proven, according to Definition 16.

When the considered wreath product is vertex-transitive (as in the cases studied in $[4,5,13])$, the graph is distance-balanced and, according to Proposition 6 , the total distance for every vertex is constant and can be easily deduced from the Wiener index by Equation (2). It is possible to check that, in these special cases, the value of the total distance via the Wiener index computation in [7] coincides with the one provided by Equation (3) in Theorem 17.

Notice that the coefficient of the term $W_{\rho_{A}}(u, G)$ in Equation (3) of Theorem 17 only depends on the cardinality of $|A|$. This motivates the following definition.

Definition 18. Let $G=\left(V_{G}, E_{G}\right)$ be a graph with $\left|V_{G}\right|=n$, and let $u \in V_{G}$. For any $k \in\{0,1, \ldots, n\}$, set

$$
W_{\rho_{k}}(u, G):=\sum_{A \subseteq V_{G},|A|=k} W_{\rho_{A}}(u, G) .
$$

Corollary 19. Let $G=\left(V_{G}, E_{G}\right)$ and $H=\left(V_{H}, E_{H}\right)$ be two graphs with $\left|V_{G}\right|=n$, $\left|V_{H}\right|=m$. Let $u=(y, x)=\left(y_{1}, \ldots, y_{n}\right) x \in V_{G i H}$. Then we have

$$
W(u, G \curlywedge H)=n m^{n-1} \sum_{i=1}^{n} W\left(y_{i}, H\right)+\sum_{k=0}^{n}(m-1)^{k} W_{\rho_{k}}(x, G) .
$$

Proof. It follows from Theorem 17 and Definition 18. 
Observe that the total distance of a vertex $u=\left(y_{1}, \ldots, y_{n}\right) x \in V_{G i H}$ depends on the total distance of the vertices $y_{i}$ in $H$ and on the quantities $W_{\rho_{k}}(x, G)$, for $k=0, \ldots, n$. This implies that computing $W_{\rho_{k}}(x, G)$ for $G$ allows to immediately deduce the total distances in any graph $G$ ? $H$, when the total distances in $H$ are known.

Remark 20. Notice that if there exists $u \in V_{G}$ such that $\rho_{V_{G}}(u, u)=n$, then $\rho_{V_{G}}(v, v)=n$ for all $v \in V_{G}$, and this is equivalent to the existence of a Hamiltonian cycle, that is, a closed walk that visits each vertex of $G$ exactly once. For any $u, v \in V_{G}, u \neq v$, we have that $\rho_{V_{G}}(u, v)=n-1$ if and only if there exists a Hamiltonian path from $u$ to $v$, that is, a walk from $u$ to $v$ visiting each vertex of $G$ exactly once. A graph $G$ is said to be Hamiltonian-connected from the vertex $u$ if a Hamiltonian path exists from $u$ to every other vertex $v \neq u$ (see [11]). Then it is easy to observe that

$$
W_{\rho_{n}}(u, G)=n^{2}-n+1 \Longleftrightarrow G \text { is Hamiltonian-connected from } u \text {. }
$$

In [11] it is stated the NP-completeness of the problem to detect those pairs $(G, u)$ such that $G$ is Hamiltonian-connected from the vertex $u$. As a consequence, the problem of computing $W_{\rho_{n}}(u, G)$ is NP-hard.

\section{Explicit computations on star graphs}

In this section, we focus our attention on a non-distance-balanced example: the star graph $S_{n}$. We start by computing the values of $W_{\rho_{k}}\left(x, S_{n}\right)$, which are necessary to analyze the total distances (and the Wiener index) in wreath products having $S_{n}$ as first factor graph. We consider $S_{n} \imath K_{m}$ in Section 4.1: in this case, the total distance takes only two distinct values. The situation becomes more interesting and rich in the case of the graph $S_{n} \prec S_{m}$, treated in Section 4.2. Both the graphs $S_{n} \prec K_{m}$ and $S_{n} \prec S_{m}$ are not distance-balanced. In the second case, we also provide some upper and lower bounds for the opportunity index $o p p\left(S_{n} \curlywedge S_{m}\right)$.

In the star graph $S_{n}=\left(V_{S_{n}}, E_{S_{n}}\right)$, for a given subset $A \subseteq V_{S_{n}}$ we define $A^{*}:=A \backslash\{c\}$, where $c$ is the center of $S_{n}$.

Since the case $A=\emptyset$ has been already discussed in Example 10, we can assume $|A| \geqslant 1$. Given any $u, v \in V_{S_{n}} \backslash\{c\}$, one can check that:

$$
\begin{aligned}
& \rho_{A}(u, v)= \begin{cases}2\left|A^{*}\right|+2 & \text { if } u, v \notin A \\
2\left|A^{*}\right| & \text { if }(u \notin A, v \in A) \text { or }(u \in A, v \notin A) \\
2\left|A^{*}\right|-2 & \text { if } u, v \in A, u \neq v \\
2\left|A^{*}\right| & \text { if } u, v \in A, u=v, A \neq\{u\} \\
0 & \text { if } u, v \in A, u=v, A=\{u\}\end{cases} \\
& \rho_{A}(u, c)= \begin{cases}2\left|A^{*}\right|+1 & \text { if } u \notin A \\
2\left|A^{*}\right|-1 & \text { if } u \in A\end{cases} \\
& \rho_{A}(c, c)=2\left|A^{*}\right| .
\end{aligned}
$$


In particular, for $u, v \in V_{S_{n}} \backslash\{c\}$, we deduce:

$$
\begin{aligned}
& d_{H a}(u, v)= \begin{cases}2 n-4 & \text { if } u \neq v \\
2 n-2 & \text { if } u=v,\end{cases} \\
& d_{H a}(u, c)=2 n-3, \\
& d_{H a}(c, c)=2 n-2 .
\end{aligned}
$$

Proposition 21. For every $u \in V_{S_{n}}$ we have

$$
e_{H a}(u)=2 n-2 \text {. }
$$

Proof. By virtue of Equation (6), for every $u \in V_{S_{n}}$, we have $e_{H a}(u)=\max \left\{d_{H a}(u, v), v \in\right.$ $\left.V_{S_{n}}\right\}=d_{H a}(u, u)=2 n-2$.

Theorem 22. Let $x \in V_{S_{n}}$. Then:

$$
W_{\rho_{k}}\left(x, S_{n}\right)= \begin{cases}2 n-3 & \text { if } k=0 \text { and } x \neq c \\
4 n^{2}-7 n+2 & \text { if } k=1 \text { and } x \neq c \\
\left(\begin{array}{c}
n-2 \\
k
\end{array}\right) f(n, k)+2\left(\begin{array}{c}
n-2 \\
k-1
\end{array}\right) f(n, k-1)+\left(\begin{array}{c}
n-2 \\
k-2
\end{array}\right) f(n, k-2) & \text { if } 1<k \leqslant n \text { and } x \neq c \\
n-1 & \text { if } k=0 \text { and } x=c \\
\left(\begin{array}{c}
n-1 \\
k
\end{array}\right) c(n, k)+\left(\begin{array}{c}
n-1 \\
k-1
\end{array}\right) c(n, k-1) & \text { if } 1 \leqslant k \leqslant n \text { and } x=c\end{cases}
$$

where

$$
f(n, k):=2 n k+2 n-2 k-1 \quad \text { and } \quad c(n, k):=f(n, k)-n .
$$

Proof. The case $k=0$ is treated in Example 10; the case $k=1$ can be studied by a direct computation, using Definition 16 and Equation (5). So assume $|A|=k>1$. We want to prove that:

$$
W_{\rho_{A}}\left(x, S_{n}\right)= \begin{cases}f\left(n,\left|A^{*} \backslash\{x\}\right|\right) & \text { if } x \neq c \\ c\left(n,\left|A^{*} \backslash\{x\}\right|\right) & \text { if } x=c .\end{cases}
$$

We are going to analyze each case by using Equation (5).

Suppose $x \neq c$.

If $x, c \notin A$, then $|A|=\left|A^{*}\right|=\left|A^{*} \backslash\{x\}\right|=k$. We get:

$$
\begin{aligned}
W_{\rho_{A}}\left(x, S_{n}\right) & =\sum_{y \in V_{S_{n}}} \rho_{A}(x, y)=\sum_{y \in A} \rho_{A}(x, y)+\sum_{y \in V_{S_{n}} \backslash(A \cup\{c\})} \rho_{A}(x, y)+\rho_{A}(x, c) \\
& =|A|(2|A|)+(n-|A|-1)(2|A|+2)+(2|A|+1) \\
& =2 k^{2}+(n-k-1)(2 k+2)+(2 k+1) \\
& =f(n, k) .
\end{aligned}
$$


If $x \in A, c \notin A$, then $|A|=\left|A^{*}\right|=k$ and $\left|A^{*} \backslash\{x\}\right|=k-1$. We get:

$$
\begin{aligned}
W_{\rho_{A}}\left(x, S_{n}\right) & =\sum_{y \in V_{S_{n}}} \rho_{A}(x, y)=\sum_{y \in A \backslash\{x\}} \rho_{A}(x, y)+\rho_{A}(x, x)+\sum_{y \in V_{S_{n}} \backslash(A \cup\{c\})} \rho_{A}(x, y)+\rho_{A}(x, c) \\
& =(|A|-1)(2|A|-2)+2|A|+(n-|A|-1)(2|A|)+(2|A|-1) \\
& =(k-1)(2 k-2)+2 k+(n-k-1) 2 k+2 k-1 \\
& =f(n, k-1) .
\end{aligned}
$$

If $x \notin A, c \in A$, then $|A|=k$ and $\left|A^{*}\right|=\left|A^{*} \backslash\{x\}\right|=k-1$. We have:

$$
W_{\rho_{A}}\left(x, S_{n}\right)=W_{\rho_{A^{*}}}\left(x, S_{n}\right)=f(n, k-1) .
$$

If $x, c \in A$, then $|A|=k,\left|A^{*}\right|=k-1$ and $\left|A^{*} \backslash\{x\}\right|=k-2$. We have:

$$
W_{\rho_{A}}\left(x, S_{n}\right)=W_{\rho_{A^{*}}}\left(x, S_{n}\right)=f(n, k-2) .
$$

For a fixed $k$ such that $1<k \leqslant n$, we have $\left(\begin{array}{c}n-2 \\ k\end{array}\right)$ subsets $A$ of cardinality $k$, such that $x \notin A, c \notin A$ (and then $\left|A^{*} \backslash\{x\}\right|=k$ ); we have $2\left(\begin{array}{c}n-2 \\ k-1\end{array}\right)$ subsets $A$ of cardinality $k$, such that $x \in A, c \notin A$ or $x \notin A, c \in A$ (and then $\left|A^{*} \backslash\{x\}\right|=k-1$ ); finally, we have $\left(\begin{array}{l}n-2 \\ k-2\end{array}\right)$ subsets $A$ of cardinality $k$, such that $x \in A, c \in A$ (and then $\left|A^{*} \backslash\{x\}\right|=k-2$ ). This gives the expression of $W_{\rho_{k}}\left(x, S_{n}\right)$ in the claim.

Suppose now $x=c$.

If $c \notin A$, then $|A|=\left|A^{*}\right|=\left|A^{*} \backslash\{x\}\right|=k$. We have:

$$
\begin{aligned}
W_{\rho_{A}}\left(c, S_{n}\right) & =\sum_{y \in V_{S_{n}}} \rho_{A}(c, y)=\sum_{y \in A} \rho_{A}(c, y)+\sum_{y \in V_{S_{n}} \backslash(A \cup\{c\})} \rho_{A}(c, y)+\rho_{A}(c, c) \\
& =|A|(2|A|-1)+(n-|A|-1)(2|A|+1)+2|A| \\
& =k(2 k-1)+(n-k-1)(2 k+1)+2 k \\
& =c(n, k) .
\end{aligned}
$$

If $c \in A,|A|=k$, then $\left|A^{*}\right|=\left|A^{*} \backslash\{x\}\right|=k-1$. We have:

$$
\begin{aligned}
W_{\rho_{A}}\left(c, S_{n}\right) & =\sum_{y \in V_{S_{n}}} \rho_{A}(c, y)=\sum_{y \in A \backslash\{c\}} \rho_{A}(c, y)+\rho_{A}(c, c)+\sum_{y \in V_{S_{n}} \backslash A} \rho_{A}(c, y) \\
& =(|A|-1)\left(2\left|A^{*}\right|-1\right)+2\left|A^{*}\right|+(n-|A|)\left(2\left|A^{*}\right|+1\right) \\
& =(k-1)(2(k-1)-1)+2(k-1)+(n-k)(2(k-1)+1) \\
& =c(n, k-1) .
\end{aligned}
$$

For a fixed $k$ such that $1 \leqslant k \leqslant n$, we have $\left(\begin{array}{c}n-1 \\ k\end{array}\right)$ subsets $A$ of cardinality $k$, such that $c \notin A$ (and then $\left|A^{*} \backslash\{x\}\right|=k$ ), we have $\left(\begin{array}{l}n-1 \\ k-1\end{array}\right)$ subsets $A$ of cardinality $k$, such that $c \in A$ (and then $\left.\left|A^{*} \backslash\{x\}\right|=k-1\right)$. This gives the expression of $W_{\rho_{k}}\left(c, S_{n}\right)$ in the claim. 


\subsection{The wreath product $S_{n} \prec K_{m}$}

The first example of wreath product that we consider is the graph $S_{n}$ 乙 $K_{m}$. As we will see in Theorem 23, the total distance of a vertex $u=\left(y_{1}, \ldots, y_{n}\right) x \in V_{S_{n} \imath K_{m}}$ in this case can only take two values, depending on the fact that $x$ (the lamplighter position) is the center of the star, or a leaf.

Before analyzing the total distances, notice that $e_{K_{m}}(y)=1$ independently on the choice of the vertex $y$ in $V_{K_{m}}$. Combining with Corollary 14 and Proposition 21, for every $u \in V_{S_{n} \imath K_{m}}$, we have

$$
e_{S_{n} 2 K_{m}}(u)=3 n-2 .
$$

Moreover, we recall that, for every vertex $y \in V_{K_{m}}$, one has $W\left(y, K_{m}\right)=m-1$ (see Example 9). Then the following result holds.

Theorem 23. Let $u=\left(y_{1}, \ldots, y_{n}\right) x \in V_{S_{n} \curlywedge K_{m}}$. We have:

$$
W\left(u, S_{n} \curlywedge K_{m}\right)= \begin{cases}m^{n-1}\left(3 m n^{2}-4 m n-3 n^{2}+3 m+6 n-4\right)-2 m & \text { if } x \neq c \\ m^{n-1}\left(3 m n^{2}-3 m n-3 n^{2}+m+4 n-2\right) & \text { if } x=c .\end{cases}
$$

Proof. We combine the formula in Corollary 19 with the computation of $W_{\rho_{k}}\left(x, S_{n}\right)$ in Theorem 22.

Corollary 24. Let $u=\left(y_{1}, \ldots, y_{n}\right) x \in V_{S_{n} \imath K_{m}}$. If $m=2$, or if $m=n=3$, the vertex $u$ is median if and only if $x=c$. In all the other cases, the vertex $u$ is median if and only if $x \neq c$.

Proof. Let $u_{c}, u_{l} \in V_{S_{n} \curlywedge K_{m}}$ be two vertices with lamplighter position at the center $c$ and at a leaf of $S_{n}$, respectively. Then

$$
\begin{aligned}
W\left(u_{c}, S_{n} \prec K_{m}\right)-W\left(u_{l}, S_{n} \prec K_{m}\right) & =m^{n-1}(m n-2 m-2 n+2)+2 m \\
& =m^{n-1}((m-2)(n-2)-2)+2 m .
\end{aligned}
$$

For $m=2$, we obtain:

$$
W\left(u_{c}, S_{n} \curlywedge K_{2}\right)-W\left(u_{l}, S_{n} \curlywedge K_{2}\right)=4-2^{n},
$$

that is negative for every $n>2$. If $m=n=3$, we have:

$$
W\left(u_{c}, S_{3} \curlywedge K_{3}\right)-W\left(u_{l}, S_{3} \succ K_{3}\right)=-3 .
$$

Finally, when $m>2$ and $\max \{m, n\}>3$, we have that the contribution $(m-2)(n-2)-2$ is non-negative, and therefore

$$
W\left(u_{c}, S_{n} \curlywedge K_{m}\right)>W\left(u_{l}, S_{n} \prec K_{m}\right) .
$$

By taking the sum of the total distance $W\left(u, S_{n} \imath K_{m}\right)$ over all vertices $u \in V_{S_{n} \imath K_{m}}$, and dividing by 2 (see Equation (2)), we deduce the expression for the Wiener index of the graph $S_{n} \prec K_{m}$. 
Corollary 25. The Wiener index of the graph $S_{n} \curlywedge K_{m}$ is

$$
W\left(S_{n} \curlywedge K_{m}\right)=m^{2 n-1}(m-1)\left(\frac{3}{2} n^{3}-1\right)+m^{n+1}(n-1)\left(3 m^{n-2} n-2 m^{n-1} n-1\right) .
$$

Proof. It follows from Theorem 23, using the fact that the number of vertices $\left(y_{1}, \ldots, y_{n}\right) x$ such that $x \neq c$ is $(n-1) m^{n}$, and the number of vertices $\left(y_{1}, \ldots, y_{n}\right) x$ such that $x=c$ is $m^{n}$.

Remark 26. In the case $n=3$, the graph $S_{3}$ is isomorphic to $P_{3}$, the path graph on 3 vertices. According to Corollary 25, we get:

$$
W\left(S_{3} \succ K_{m}\right)=m^{4}\left(\frac{55}{2} m^{2}-\frac{43}{2} m-2\right) .
$$

Notice that we obtain the same value by using the results in [7] for the graph $P_{3}$.

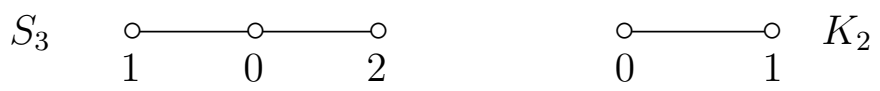

Figure 2: The graphs $S_{3}$ and $K_{2}$.

Example 27. Consider the graphs $S_{3}$ and $K_{2}$ in Fig. 2, and put $V_{S_{3}}=\{0,1,2\}$ and $V_{K_{2}}=\{0,1\}$. Then the wreath product $S_{3} 2 K_{2}$ is presented in Fig. 3. By virtue of Corollary 24, the median vertices are $M\left(S_{3} \prec K_{2}\right)=\left\{\left(y_{1}, y_{2}, y_{3}\right) 0: y_{i} \in\{0,1\}\right\}$; they are emphasized in black in the picture.

\subsection{The wreath product $S_{n} \prec S_{m}$}

In this section, we compute the total distances in the graph $S_{n}<S_{m}$ and we give an approximation of the opportunity index for these graphs, by presenting a lower and an upper bound in terms of tail conditional expectations of the binomial distribution with probability $p=\frac{m-1}{m}$.

We will use the same notation $c$ in order to denote the central vertex of the first graph and the central vertex of the second graph, being unlikely to cause confusion. For a vertex $u=\left(y_{1}, \ldots, y_{n}\right) x \in V_{S_{n} S_{m}}$ let us put $l(u):=\left|\left\{i \in\{1, \ldots, n\}: y_{i} \neq c\right\}\right|$, that is, $l(u)$ is the number of leaves in the lamp configuration of $u$.

Before analyzing the total distances, notice that in $S_{m}$ one has $e_{S_{m}}(c)=1$ and $e_{S_{m}}(l)=$ 2 if $l \in V_{S_{m}}$ is a leaf. Combining with Corollary 14 and Proposition 21, for every $u \in$ $S_{n} \prec K_{m}$ we have

$$
e_{S_{n} 2 S_{m}}(u)=3 n+l(u)-2 .
$$

In particular the vertices $(c, \ldots, c) x$, for every $x \in V_{S_{n}}$, minimize the eccentricity and are centers in $S_{n} \prec S_{m}$; the diameter is $\operatorname{diam}\left(S_{n} \prec S_{m}\right)=4 n-2$. 


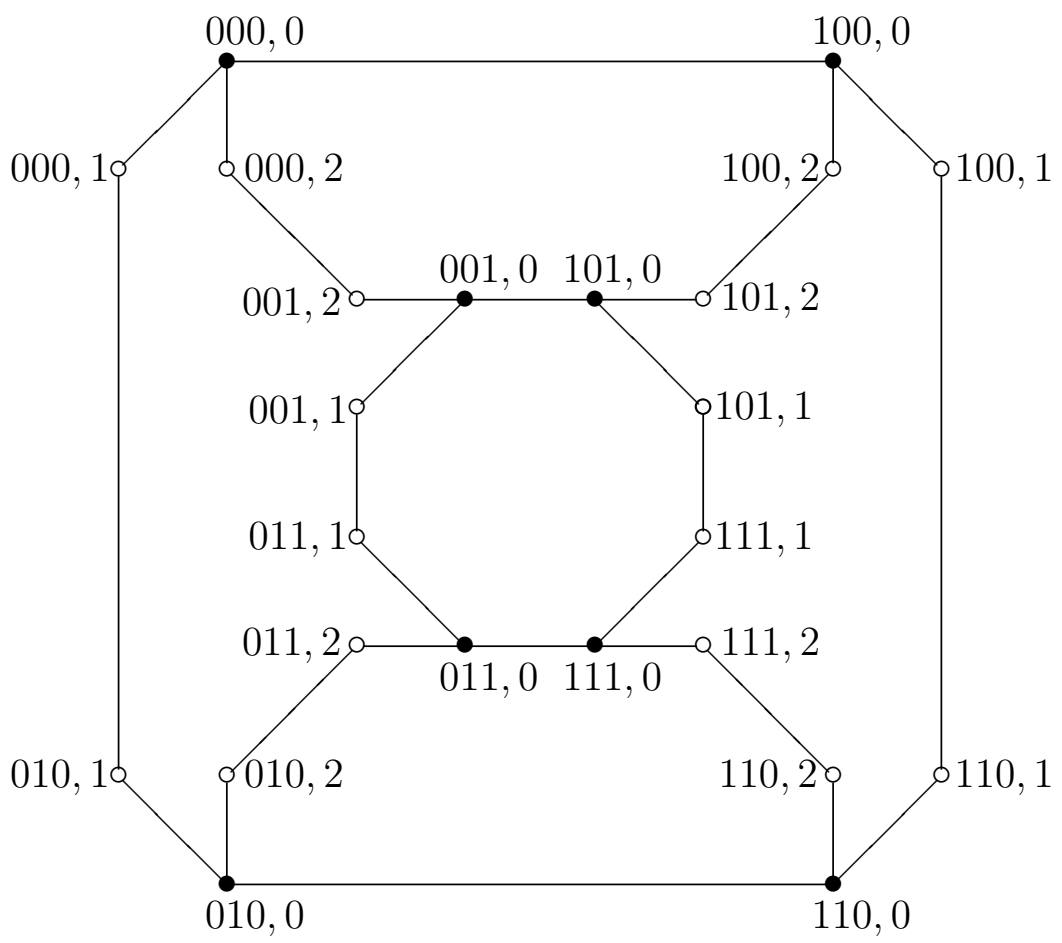

Figure 3: The graph $S_{3}$ 々 $K_{2}$.

Theorem 28. Let $u=\left(y_{1}, \ldots, y_{n}\right) x \in V_{S_{n} \backslash S_{m}}$. Then we have:

$$
W\left(u, S_{n} \prec S_{m}\right)= \begin{cases}m^{n-1}\left[3 m-3 n^{2}-4 m n+3 m n^{2}+6 n-4+n(m-2) l(u)\right]-2 m & \text { if } x \neq c \\ m^{n-1}\left[m-3 n^{2}-3 m n+3 m n^{2}+4 n-2+n(m-2) l(u)\right] & \text { if } x=c .\end{cases}
$$

Proof. Recall from Example 10 that in $S_{m}$,

$$
W\left(y, S_{m}\right)= \begin{cases}m-1 \text { if } & y=c \\ 2 m-3 \text { if } & y \neq c\end{cases}
$$

Then we have to combine Equation (4) with the computation of the $W_{\rho_{k}}\left(x, S_{n}\right)$ given in Theorem 22 .

It is possible to observe that the difference between the total distances of two vertices $u=(y, x), u^{\prime}=\left(y^{\prime}, x^{\prime}\right) \in V_{S_{n} \imath S_{m}}$ only depends on the difference between the number of leaves in their lamp configuration (i.e., on $\left.l(u)-l\left(u^{\prime}\right)\right)$ and on the positions $x$ and $x^{\prime}$ of the lamplighter; on the other hand, the quantity $W\left(u, S_{n} \prec S_{m}\right)-W\left(u^{\prime}, S_{n} \prec S_{m}\right)$ does not depend on the particular values of $l(u)$ and $l\left(u^{\prime}\right)$. If we set:

$$
\begin{aligned}
& W_{\min }:=m^{n-1}\left(3 m-3 n^{2}-4 m n+3 m n^{2}+6 n-4\right)-2 m, \\
& \Delta:=m^{n-1} n(m-2), \\
& \Delta_{l c}:=m^{n-1}(m n-2 m-2 n+2)+2 m,
\end{aligned}
$$


we obtain

$$
W\left(u, S_{n} \prec S_{m}\right)= \begin{cases}W_{\text {min }}+l(u) \Delta & \text { if } x \neq c \\ W_{\text {min }}+l(u) \Delta+\Delta_{l c} & \text { if } x=c .\end{cases}
$$

Corollary 29. If $\max \{n, m\}>3$, a vertex $u=\left(y_{1}, \ldots, y_{n}\right) x \in S_{n} 2 S_{m}$ is a median vertex if and only if $x \neq c$ and $l(u)=0$. That is, $M\left(S_{n} \prec S_{m}\right)=\left\{(c, \ldots, c) x: x \in V_{S_{n}}, x \neq c\right\}$.

Proof. Notice that, from the proof of Corollary 24, we have that $\Delta_{l c}$ is positive for $\max \{n, m\}>3$. Moreover, $\Delta$ is positive whenever $m>2$. By virtue of Equation (7) we can say that $u=(y, x) \in V_{S_{n} \imath S_{m}}$ is median if and only if $W\left(u, S_{n} \imath S_{m}\right)=W_{\text {min }}$, and this is possible only when $x \neq c$ and $l(u)=0$.

Example 30. The graph $S_{3} 2 S_{3}$ is represented in Fig. 4. This is the only case of wreath product $S_{n} 2 S_{m}$ such that the total distance of vertices when the lamplighter is in a leaf position is greater than the total distance of vertices when he is in the center position. We have:

$$
V_{S_{3} S_{3}}=81, \quad W_{\min }=363, \quad \Delta=27, \quad \Delta_{l c}=-3 .
$$

In this case $W_{\min }$ is not the minimal value for the total distance, because the vertex $u_{0}:=(c, c, c) c$ satisfies $W\left(u_{0}, S_{3} \succ S_{3}\right)=360$ and $M\left(S_{3} \succ S_{3}\right)=\left\{u_{0}\right\}$. The maximal value for the total distance is 444 , provided by vertices $u=\left(y_{1}, y_{2}, y_{3}\right) x$ for which $x \neq c$ and $l(u)=3$.

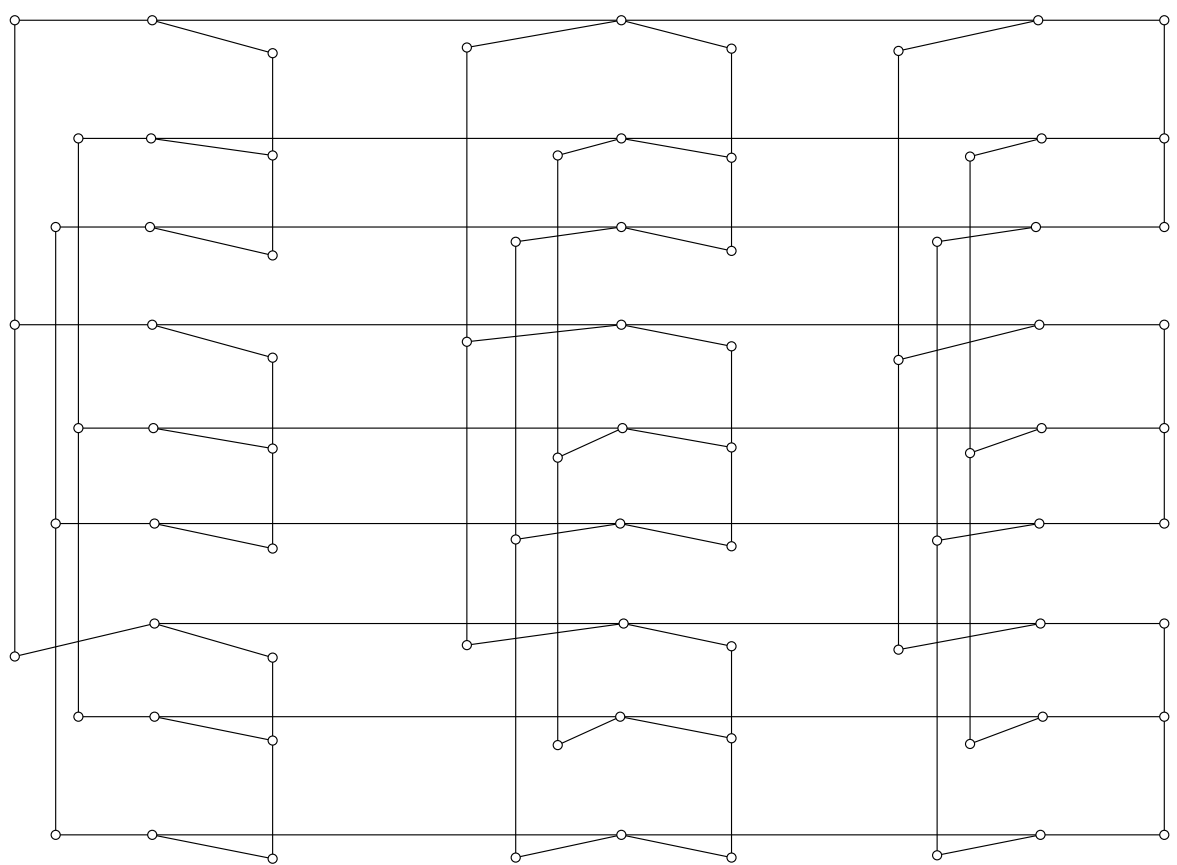

Figure 4: The graph $S_{3} 2 S_{3}$. 
For every $k=0,1, \ldots, n$, let us set:

$$
\begin{aligned}
& Z_{k}:=\left\{u \in V_{S_{n} 2 S_{m}}: l(u)=k\right\}, \\
& L_{k}:=\left\{\left(y_{1}, \ldots, y_{n}\right) x \in Z_{k}: x \neq c\right\}, \\
& C_{k}:=\left\{\left(y_{1}, \ldots, y_{n}\right) x \in Z_{k}: x=c\right\} .
\end{aligned}
$$

Notice that $Z_{k}=L_{k} \sqcup C_{k}$, for each $k$. Moreover, it is easy to check that:

$$
\left|Z_{k}\right|=n\left(\begin{array}{l}
n \\
k
\end{array}\right)(m-1)^{k} ; \quad\left|L_{k}\right|=(n-1)\left(\begin{array}{l}
n \\
k
\end{array}\right)(m-1)^{k} ; \quad\left|C_{k}\right|=\left(\begin{array}{l}
n \\
k
\end{array}\right)(m-1)^{k} .
$$

We can observe that the number of lamp coordinates that are leaves of a random vertex of $S_{n} 2 S_{m}$ follows the binomial distribution $B(n, p)$ with parameter $n$ and $p:=\frac{m-1}{m}$. Probability $p$ is in fact the probability for a coordinate to be a leaf, and the coordinates are independent. Then we can consider a random variable $X_{p}$ such that $X_{p} \sim B(n, p)$, where

$$
P\left(X_{p}=k\right)=\frac{\left|Z_{k}\right|}{\left|V_{S_{n} S_{m}}\right|}=\frac{n\left(\begin{array}{l}
n \\
k
\end{array}\right)(m-1)^{k}}{n m^{n}}=\left(\begin{array}{l}
n \\
k
\end{array}\right) p^{k}(1-p)^{n-k} .
$$

Note that $1-p=\frac{1}{m}$.

Remark 31. The following are well known in probability theory (see [14]):

- $E\left(X_{p}\right)=\sum_{i=0}^{n}\left(\begin{array}{c}n \\ i\end{array}\right) p^{i}(1-p)^{n-i} i=n p=\frac{n(m-1)}{m} ;$

- $E\left(X_{p} \mid X_{p} \leqslant k\right)=\frac{\sum_{i=0}^{k}\left(\begin{array}{l}n \\ i\end{array}\right) p^{i}(1-p)^{n-i} i}{\sum_{i=0}^{k}\left(\begin{array}{c}n \\ i\end{array}\right) p^{i}(1-p)^{n-i}} ;$

- $E\left(X_{p} \mid X_{p} \geqslant k\right)=\frac{\sum_{i=k}^{n}\left(\begin{array}{c}n \\ i\end{array}\right) p^{i}(1-p)^{n-i} i}{\sum_{i=k}^{n}\left(\begin{array}{c}n \\ i\end{array}\right) p^{i}(1-p)^{n-i}}$.

Corollary 32. The Wiener index of the graph $S_{n} \prec S_{m}$ is

$$
W\left(S_{n} \curlywedge S_{m}\right)=\frac{n m^{n}}{2}\left(W_{\min }+\frac{1}{n} \Delta_{l c}+\Delta \frac{(m-1) n}{m}\right) .
$$

Proof. By summing the total distances $W\left(u, S_{n} \prec S_{m}\right)$ computed in Theorem 28 over all vertices $u \in V_{S_{n} 2 S_{m}}$, and dividing by 2 (see Equation (2)), we obtain:

$$
\begin{aligned}
W\left(S_{n} \imath S_{m}\right) & =\frac{1}{2} \sum_{i=0}^{n}\left(\left|C_{i}\right|\left(W_{\text {min }}+i \Delta+\Delta_{l c}\right)+\left|L_{i}\right|\left(W_{\text {min }}+i \Delta\right)\right) \\
& =\frac{\left|V_{S_{n} 2 S_{m}}\right|}{2\left|V_{S_{n} 2 S_{m}}\right|} \sum_{i=0}^{n}\left(\left|C_{i}\right|\left(W_{\text {min }}+i \Delta+\Delta_{l c}\right)+\left|L_{i}\right|\left(W_{\text {min }}+i \Delta\right)\right) \\
& =\frac{\left|V_{S_{n} 2 S_{m}}\right|}{2}\left(W_{\text {min }}+\frac{1}{n} \Delta_{l c}+\Delta \frac{n \sum_{i=0}^{n}\left(\begin{array}{c}
n \\
i
\end{array}\right)(m-1)^{i} i}{n m^{n}}\right) \\
& =\frac{\left|V_{S_{n} 2 S_{m}}\right|}{2}\left(W_{\min }+\frac{1}{n} \Delta_{l c}+\Delta E\left(X_{p}\right)\right) .
\end{aligned}
$$


In the remaining part of the paper we will give an estimate of the opportunity index of the graph $S_{n} 2 S_{m}$. At first we present some general results and observations. The following lemma is contained in the proof of Therem 3.1 in [3].

Lemma 33. Let $G=\left(V_{G}, E_{G}\right)$ be a graph and consider the partition $V_{G}=A \sqcup B$. Then:

$$
W_{A}(G)-W_{B}(G)=\frac{1}{2}(W(A, G)-W(B, G)),
$$

where $W(A, G):=\sum_{u \in A} W(u, G)$, and $W_{A}(G)$ is as in Equation (1).

Proof. It suffices to compute the difference between

$$
W(A, G)=\sum_{u \in A} W(u, G)=\sum_{u \in A, v \in A} d_{G}(u, v)+\sum_{u \in A, v \in B} d_{G}(u, v)=2 W_{A}(G)+\sum_{u \in A, v \in B} d_{G}(u, v),
$$

and

$W(B, G)=\sum_{u \in B} W(u, G)=\sum_{u \in B, v \in B} d_{G}(u, v)+\sum_{u \in B, v \in A} d_{G}(u, v)=2 W_{B}(G)+\sum_{u \in A, v \in B} d_{G}(u, v)$.

Definition 34. Let $G=\left(V_{G}, E_{G}\right)$ be a graph, and let $A, B \subseteq V_{G}$. We write

$$
B \preceq A,
$$

if $\forall u \in A, \forall v \in B$, we have $W(v, G) \leqslant W(u, G)$.

Lemma 35. Let $G=\left(V_{G}, E_{G}\right)$ be a graph and let $V_{1}, V_{2}, V_{3} \subseteq V_{G}$ such that $V_{1}=V_{2} \sqcup V_{3}$, and $V_{3} \preceq V_{2}$. Then $\frac{W\left(V_{2}, G\right)}{\left|V_{2}\right|} \geqslant \frac{W\left(V_{1}, G\right)}{\left|V_{1}\right|}$.

Proof. The average on a subset of values greater than the others is greater than the average on all values.

Proposition 36. Let $G=\left(V_{G}, E_{G}\right)$ be a graph. Suppose $V_{G}=A \sqcup B$, with $|A|=|B|$, and $B \preceq A$. Then we have

$$
\text { opp }(G)=\frac{1}{2}(W(A, G)-W(B, G))=W(A, G)-W(G)=W(G)-W(B, G) .
$$

Proof. By virtue of Lemma 33

$$
\operatorname{opp}(G)=\max \left\{\frac{1}{2}\left(W\left(V_{1}, G\right)-W\left(V_{2}, G\right)\right):\left\{V_{1}, V_{2}\right\} \text { is a half-partition of } V_{G}\right\}
$$

and it is clear that the partition $\{A, B\}$ realizes the maximum since $B \preceq A$ by assumption. The other two equalities easily follow from the fact that $2 W(G)=W(A, G)+W(B, G)$. 
Coming back to the graph $S_{n} \prec S_{m}$, we are going to approximate the sets $A$ and $B$ of Proposition 36 by the following sets, with a suitable choice of $k$ :

$$
A_{k}:=\left\{u \in V_{S_{n} 2 S_{m}}: l(u) \geqslant k\right\}, \quad B_{k}:=\left\{u \in V_{S_{n} 2 S_{m}}: l(u) \leqslant k\right\} .
$$

Notice that $\Delta-\Delta_{l c}=2 m\left(m^{n-1}-m^{n-2}-1\right)>0$. Therefore, by virtue of Equation (7), if $\max \{n, m\}>3$, we have:

$$
L_{k} \preceq C_{k} \preceq L_{k+1} .
$$

From now on we will focus on the case $\max \{n, m\}>3$. Let us denote with $d_{p}$ the median of $X_{p}$ : by definition, we have

$$
d_{p}=\min \left\{k \in\{0,1, \ldots, n\}: \sum_{i=0}^{k} P\left(X_{p}=i\right) \geqslant \frac{1}{2}\right\} .
$$

It follows that

$$
\begin{aligned}
d_{p} & =\min \left\{k \in\{0,1, \ldots, n\}: \sum_{i=0}^{k}\left|Z_{i}\right| \geqslant \frac{\left|V_{S_{n} 2 S_{m}}\right|}{2}\right\} \\
& =\max \left\{k \in\{0,1, \ldots, n\}:\left|A_{k}\right| \geqslant \frac{\left|V_{S_{n} 2 S_{m}}\right|}{2}\right\} \\
& =\min \left\{k \in\{0,1, \ldots, n\}:\left|B_{k}\right| \geqslant \frac{\left|V_{S_{n} \imath S_{m}}\right|}{2}\right\} .
\end{aligned}
$$

Lemma 37. If $V_{S_{n} 2 S_{m}}=A \sqcup B$, with $B \preceq A$ and $|A|=|B|$, then:

$$
\begin{aligned}
& \frac{W\left(A_{d_{p}}, S_{n} \curlywedge S_{m}\right)}{\left|A_{d_{p}}\right|} \leqslant \frac{W\left(A, S_{n} \curlywedge S_{m}\right)}{|A|} \leqslant \frac{W\left(A_{d_{p}+1}, S_{n} \curlywedge S_{m}\right)}{\left|A_{d_{p}+1}\right|}, \\
& \frac{W\left(B_{d_{p}-1}, S_{n} \curlywedge S_{m}\right)}{\left|B_{d_{p}-1}\right|} \leqslant \frac{W\left(B, S_{n} \curlywedge S_{m}\right)}{|B|} \leqslant \frac{W\left(B_{d_{p}}, S_{n} \curlywedge S_{m}\right)}{\left|B_{d_{p}}\right|} .
\end{aligned}
$$

Proof. By the hypothesis $B \preceq A$ and by (9), we can write $A=A_{d_{p}+1} \sqcup C$, with $C \preceq A_{d_{p}+1}$. Then by virtue of Lemma 35, we have

$$
\frac{W\left(A_{d_{p}+1}, S_{n} \curlywedge S_{m}\right)}{\left|A_{d_{p}+1}\right|} \geqslant \frac{W\left(A, S_{n} \curlywedge S_{m}\right)}{|A|} .
$$

Similarly $A_{d_{p}}=A \sqcup C^{\prime}$, with $C^{\prime} \preceq A$; therefore, by virtue of Lemma 35 , we have

$$
\frac{W\left(A, S_{n} \curlywedge S_{m}\right)}{|A|} \geqslant \frac{W\left(A_{d_{p}}, S_{n} \curlywedge S_{m}\right)}{\left|A_{d_{p}}\right|}
$$

The second part of the claim is obtained by applying the same argument for $B$, as we can write $B=B_{d_{p}-1} \sqcup D$, with $B_{d_{p}-1} \preceq D$, and $B_{d_{p}}=B \sqcup D^{\prime}$, with $B \preceq D^{\prime}$. 
Theorem 38. Consider the graph $S_{n} 2 S_{m}$ and denote by $V$ its vertex set. Then the following inequalities hold:

$$
\begin{aligned}
& \frac{m-2}{2 m}|V|^{2}\left(E\left(X_{p}\right)-E\left(X_{p} \mid X_{p} \leqslant d_{p}\right)\right) \leqslant \operatorname{opp}\left(S_{n} \imath S_{m}\right) \leqslant \frac{m-2}{2 m}|V|^{2}\left(E\left(X_{p}\right)-E\left(X_{p} \mid X_{p}<d_{p}\right)\right) ; \\
& \frac{m-2}{2 m}|V|^{2}\left(E\left(X_{p} \mid X_{p} \geqslant d_{p}\right)-E\left(X_{p}\right)\right) \leqslant \operatorname{opp}\left(S_{n} \imath S_{m}\right) \leqslant \frac{m-2}{2 m}|V|^{2}\left(E\left(X_{p} \mid X_{p}>d_{p}\right)-E\left(X_{p}\right)\right) ; \\
& \frac{m-2}{4 m}|V|^{2}\left(E\left(X_{p} \mid X_{p} \geqslant d_{p}\right)-E\left(X_{p} \mid X_{p} \leqslant d_{p}\right)\right) \leqslant \\
& \operatorname{opp}\left(S_{n} \imath S_{m}\right) \leqslant \\
& \frac{m-2}{4 m}|V|^{2}\left(E\left(X_{p} \mid X_{p}>d_{p}\right)-E\left(X_{p} \mid X_{p}<d_{p}\right)\right) .
\end{aligned}
$$

Proof. From (10) and by using Proposition 36, we have:

$$
\begin{aligned}
& W\left(S_{n} \curlywedge S_{m}\right)-\frac{|B| W\left(B_{d_{p}}, S_{n} \curlywedge S_{m}\right)}{\left|B_{d_{p}}\right|} \leqslant \operatorname{opp}\left(S_{n} \curlywedge S_{m}\right) \leqslant W\left(S_{n} \curlywedge S_{m}\right)-\frac{|B| W\left(B_{d_{p}-1}, S_{n} \curlywedge S_{m}\right)}{\left|B_{d_{p}-1}\right|} \\
& \frac{|A| W\left(A_{d_{p}}, S_{n} \prec S_{m}\right)}{\left|A_{d_{p}}\right|}-W\left(S_{n} \curlywedge S_{m}\right) \leqslant \operatorname{opp}\left(S_{n} \curlywedge S_{m}\right) \leqslant \frac{|A| W\left(A_{d_{p}+1}, S_{n} \prec S_{m}\right)}{\left|A_{d_{p}+1}\right|}-W\left(S_{n} \curlywedge S_{m}\right) \text {. }
\end{aligned}
$$

Notice that $W\left(A_{k}, S_{n} \curlywedge S_{m}\right)=\sum_{i=k}^{n}\left(\left|C_{i}\right|\left(W_{\min }+i \Delta+\Delta_{l c}\right)+\left|L_{i}\right|\left(W_{\min }+i \Delta\right)\right)$ and $W\left(B_{k}, S_{n} \curlywedge S_{m}\right)=\sum_{i=0}^{k}\left(\left|C_{i}\right|\left(W_{\min }+i \Delta+\Delta_{l c}\right)+\left|L_{i}\right|\left(W_{\min }+i \Delta\right)\right)$. Let us focus on the first inequality in (11). By similar computations as in Corollary 32, we have:

$$
\begin{aligned}
\frac{W\left(B_{k}, S_{n} \curlywedge S_{m}\right)}{\left|B_{k}\right|} & =\frac{\sum_{i=0}^{k}\left(\left|C_{i}\right|\left(W_{\min }+i \Delta+\Delta_{l c}\right)+\left|L_{i}\right|\left(W_{\min }+i \Delta\right)\right)}{\sum_{i=0}^{k}\left|Z_{i}\right|} \\
& =\frac{\sum_{i=0}^{k}\left(\left|Z_{i}\right|\left(W_{\min }+i \Delta\right)+\left|C_{i}\right| \Delta_{l c}\right)}{\sum_{i=0}^{k}\left|Z_{i}\right|} \\
& =W_{\text {min }}+\frac{1}{n} \Delta_{l c}+\Delta \frac{\sum_{i=0}^{k} n\left(\begin{array}{c}
n \\
i
\end{array}\right)(m-1)^{i} i}{\sum_{i=0}^{k} n\left(\begin{array}{c}
n \\
i
\end{array}\right)(m-1)^{i}} \\
& =W_{\text {min }}+\frac{1}{n} \Delta_{l c}+\Delta \frac{\sum_{i=0}^{k}\left(\begin{array}{c}
n \\
i
\end{array}\right)\left(\frac{m-1}{m}\right)^{i}\left(\frac{1}{m}\right)^{n-i} i}{\sum_{i=0}^{k}\left(\begin{array}{c}
n \\
i
\end{array}\right)\left(\frac{m-1}{m}\right)^{i}\left(\frac{1}{m}\right)^{n-i}} .
\end{aligned}
$$

Therefore, from Equation (8), combined with Remark 31, in the case $k=d_{p}$ we have:

$$
\begin{aligned}
& W\left(S_{n} \curlywedge S_{m}\right)-\frac{|B| W\left(B_{d_{p}}, S_{n} \imath S_{m}\right)}{\left|B_{d_{p}}\right|}= \\
& =\frac{\left|V_{S_{n} \imath S_{m}}\right|}{2}\left(W_{\text {min }}+\frac{1}{n} \Delta_{l c}+\Delta E\left(X_{p}\right)\right)-|B|\left(W_{\text {min }}+\frac{1}{n} \Delta_{l c}+\Delta E\left(X_{p} \mid X_{p} \leqslant d_{p}\right)\right) \\
& =|B| \Delta\left(E\left(X_{p}\right)-E\left(X_{p} \mid X_{p} \leqslant d_{p}\right)\right) .
\end{aligned}
$$

Analogous computations for $B_{d_{p}-1}, A_{d_{p}}, A_{d_{p}+1}$, combined with (11), prove the first two chains of inequalities in the claim, since we have $|A|=|B|=\frac{n m^{n}}{2}$ and $\Delta=m^{n-1} n(m-2)$. The third one is the average of the first two. 
Remark 39. The introduction of the random variable $X_{p}$ is convenient because the binomial distribution is well studied and there is a rich literature about the estimations of the tail conditional expectation $E\left(X_{p} \mid X_{p} \leqslant k\right.$ ) (see, e.g., [14, 26]).

Example 40. Consider the graph $S_{6} \prec S_{3}$. We have:

$$
V_{S_{6} 2 S_{3}}=4374, \quad W_{\min }=44949, \quad \Delta=1458, \quad \Delta_{l c}=492 .
$$

The probability is $p=\frac{m-1}{m}=\frac{2}{3}$, then $E\left(X_{p}\right)=4$ and it is possible to check that $d_{p}=4$. Moreover we have:

$$
\begin{array}{ll}
E\left(X_{p} \mid X_{p}<d_{p}\right) \approx 2.6266, & E\left(X_{p} \mid X_{p}>d_{p}\right) \approx 5.25, \\
E\left(X_{p} \mid X_{p} \leqslant d_{p}\right) \approx 3.3235, & E\left(X_{p} \mid X_{p} \geqslant d_{p}\right) \approx 4.6451 .
\end{array}
$$

By virtue of Theorem 38 we have:

$$
2157150 \leqslant \operatorname{opp}\left(S_{6} 2 S_{3}\right) \leqslant 4379162 ; \quad 2057168 \leqslant \operatorname{opp}\left(S_{6} 2 S_{3}\right) \leqslant 3985807,
$$

and the best from the two is:

$$
2157150 \leqslant \operatorname{opp}\left(S_{6} 2 S_{3}\right) \leqslant 3985807 \text {. }
$$

Consider $A, B \subseteq V_{S_{6} S_{3}}$ such that $V_{S_{6} S_{3}}=A \sqcup B,|A|=|B|=2187$ and $B \preceq A$. It is easy to see that $B=B_{3} \sqcup P_{B}$ and $A=A_{5} \sqcup P_{A}$, with $P_{A} \cup P_{B}=Z_{4}$. Since $|B|-\left|B_{3}\right|=789 \leqslant 1200=\left|L_{4}\right|$, then $P_{B}$ contains only vertices with leaf-position and then $P_{A}$ contains 411 vertices with leaf-position and 240 vertices with center-position. Combining with Equation (7) we have:

$$
W\left(A, S_{6} 2 S_{3}\right)=114101439 ; \quad W\left(B, S_{6} \prec S_{3}\right)=108373323 .
$$

Finally $\operatorname{opp}\left(S_{6} 2 S_{3}\right)=2864058$.

\section{Conclusions and final remarks}

The complexity of the computation of total distances in a graph is polynomial in the number of vertices. However, in the case of the wreath product $G$ < $H$, if $\left|V_{G}\right|=n$, the number of vertices is exponential in $n$. On the other hand, the computation of $W_{\rho_{n}}(u, G)$ is NP-hard (see Remark 20). Nevertheless, Theorem 17 and Corollary 19 are fruitful when:

- one is interested in computing Wiener index or total distances in a family of wreath products with fixed first factor $G$;

- some properties of $G$ make the problem of computing $W_{\rho_{k}}(u, G)$ easier. 
A line for future research is to individuate those families of graphs $G$ where it is possible to obtain, if not even a closed formula, at least a fast algorithm for computing $W_{\rho_{k}}(u, G)$. As a generalization of the family of star graphs, the next candidate can be the family of more general trees. Another class of graphs, of completely different nature, is given by cycles: the high symmetry would be a help for the computation of $W_{\rho_{k}}(u, G)$. Another direction that is of interest for its potential applications is the generalization to the weighted graphs.

From a theoretical point of view, the analysis of the total distances in the wreath product is the first step to understand the distance-balanced property of a wreath product. Having necessary or sufficient conditions on the factors (for the distance-balance of the product) would produce many new examples and counterexamples, not obtained via the four classical products, for many centrality problems (see, e.g., [2, 20, 22]). In fact, the wreath product has the nice property of preserving regularity, vertex-transitivity, bipartitedness, and connectedness. This way, starting with few small examples, one can produce infinite families of non-isomorphic graphs with prescribed properties.

\section{Acknowledgements}

We wish to express our deepest gratitude to the anonymous referees for their useful comments and suggestions.

\section{References}

[1] R. A. Bailey, C. E. Praeger, C. A. Rowley, and T. P. Speed. Generalized wreath products of permutation groups. Proc. London Math. Soc., 47(3):69-82, 1983.

[2] K. Balakrishnan, M. Changat, I. Peterin, S. Špacapan, P. Šparl, and A. R. Subhamathi. Strongly distance-balanced graphs and graph products. European J. Combin., 30:1048-1053, 2009.

[3] K. Balakrishnan, B. Brešar, M. Changat, S. Klavžar, A. Vesel, and P. Žigert Pleteršek. Equal opportunity networks, distance-balanced graphs, and Wiener game. Discrete Optim., 12:150-154, 2014.

[4] F. Belardo, M. Cavaleri, and A. Donno. Wreath product of a complete graph with a cyclic graph: topological indices and spectrum. Appl. Math. Comput., 336:288-300, 2018.

[5] F. Belardo, M. Cavaleri, and A. Donno. Spectral analysis of the wreath product of a complete graph with a Cocktail Party graph. Atti Accad. Peloritana Pericolanti, Cl. Sci. Fis. Mat. Natur., 96(S2,A1), 2018.

[6] S. Cabello, and P. Lukšič. The complexity of obtaining a distance-balanced graph. Electron. J. Combin., 18(1), \#P49, 2011.

[7] M. Cavaleri, and A. Donno. Some degree and distance-based invariants of wreath products of graphs, arXiv:1805.08989. Submitted.

[8] D. D'Angeli, and A. Donno. Wreath product of matrices. Linear Algebra Appl., 513:276-303, 2017. 
[9] P. Dankelmann, and R. Entringer. Average distance, minimum degree, and spanning trees. J. Graph Theory, 33(1):1-13, 2000.

[10] P. Dankelmann. Average distance in weighted graphs. Discrete Math., 312(1):12-20, 2012.

[11] A. M. Dean, C. J. Knickerbocker, P. F. Lock, and M. Sheard. A survey of graphs Hamiltonian-connected from a vertex. Graph theory, combinatorics, and applications, Vol. 1 (Kalamazoo, MI, 1988), 297-313. Wiley-Interscience Publishers, Wiley New York, 1991.

[12] A. Donno. Generalized wreath products of graphs and groups. Graphs Combin., 31(4):915-926, 2015.

[13] A. Donno. Spectrum, distance spectrum, and Wiener index of wreath products of complete graphs. Ars Math. Contemp., 13(1):207-225, 2017.

[14] W. Feller. An introduction to probability theory and its applications. Vol. 2, Second edition, Wiley Series in probability and Statistics, 1991.

[15] R. Hammack, W. Imrich, and S. Klavžar. Handbook of product graphs. Second edition. Discrete Mathematics and its Applications (Boca Raton). CRC Press, Boca Raton, FL, xviii + 518 pp., 2011.

[16] K. Handa. Bipartite graphs with balanced (a,b)-partitions. Ars Combin. 51:113-119, 1999.

[17] O. Kariv, and S. L. Hakimi. An algorithmic approach to network location problems. I: The p-centers. SIAM J. Appl. Math., 37(3):513-538, 1979.

[18] O. Kariv, and S. L. Hakimi. An algorithmic approach to network location problems. I: The p-median. SIAM J. Appl. Math., 37(3):539-560, 1979.

[19] H. Kharazi, and E. Pourhadi. Graph theory in distribution and transportation problems and the connection to distance-balanced graphs. International Journal Series in Multidisciplinary Research, 1(3):17-30, 2015.

[20] A. Ilić, S. Klavžar, and M. Milanović. On distance-balanced graphs. European J. Combin., 31:733-737, 2010.

[21] J. Jerebic, S. Klavžar, and D. F. Rall. Distance-balanced graphs. Ann. Combin. 12:71-79, 2008.

[22] K. Kutnar, A. Malnič, D. Marušič, and Š. Miklavič. Distance-balanced graphs: symmetry conditions. Discrete Math., 306:1881-1894, 2006.

[23] J. D. P. Meldrum. Wreath products of groups and semigroups, Pitman Monographs and Surveys in Pure and Applied Mathematics, 74, Chapman and Hall/CRC, 336 pp., 1995.

[24] K. B. Reid. Balance vertices in trees. Centrality concepts in network location. Networks, 34(4):264-271, 1999.

[25] K. B. Reid. Centrality measures in trees. Advances in interdisciplinary applied discrete mathematics, 167-197, Interdiscip. Math. Sci. 11, World Sci. Publ., Hackensack, NJ, 2011. 
[26] S. M. Ross. Introduction to probability models. Eleventh edition. Elsevier/Academic Press, Amsterdam, xvi+767 pp., 2014.

[27] N. Trinajstić. Chemical graph theory, Second Edition. Mathematical Chemistry Series. CRC Press, Boca Raton, FL, xviii + 322 pp., 1992.

[28] H. Wiener. Structural determination of paraffin boiling points. J. Amer. Chem. Soc., 69:17-20, 1947.

[29] W. Woess. A note on the norms of transition operators on lamplighter graphs and groups. Internat. J. Algebra Comput., 15(5-6):1261-1272, 2015. 